\title{
ARTIFICIAL NEURAL NETWORK FOR MODELS OF HUMAN OPERATOR
}

\author{
MaRTin RŮŽEK
}

\author{
Czech Institute of Informatics, Robotics and Cybernetics (CIIRC), Czech Technical University in Prague, \\ Zikova 4, 16636 Prague 6, Czech Republic \\ correspondence: martin.ruzek@cvut.cz
}

\begin{abstract}
This paper presents a new approach to mental functions modeling with the use of artificial neural networks. The artificial neural networks seems to be a promising method for the modeling of a human operator because the architecture of the ANN is directly inspired by the biological neuron. On the other hand, the classical paradigms of artificial neural networks are not suitable because they simplify too much the real processes in biological neural network.

The search for a compromise between the complexity of biological neural network and the practical feasibility of the artificial network led to a new learning algorithm. This algorithm is based on the classical multilayered neural network; however, the learning rule is different. The neurons are updating their parameters in a way that is similar to real biological processes. The basic idea is that the neurons are competing for resources and the criterion to decide which neuron will survive is the usefulness of the neuron to the whole neural network. The neuron is not using "teacher" or any kind of superior system, the neuron receives only the information that is present in the biological system.

The learning process can be seen as searching of some equilibrium point that is equal to a state with maximal importance of the neuron for the neural network. This position can change if the environment changes. The name of this type of learning, the homeostatic artificial neural network, originates from this idea, as it is similar to the process of homeostasis known in any living cell. The simulation results suggest that this type of learning can be useful also in other tasks of artificial learning and recognition.
\end{abstract}

KEYwORDS: neural network, artificial neuron, learning algorithm, mental model.

\section{INTRODUCTION}

For many practical applications it would be useful to have a model of a human operator, as for example in transport engineering where the human operator plays a principal role. Unfortunately, the human factor causes also most of the accidents; therefore it would be helpful to understand the processes in the human brain. Due to the complex nature of the human brain it is however very difficult to model and predict its behavior.

he need to dispose of the model of a human operator, as the most complex part of many transport systems, stays behind this research. The transport and traffic simulations are on high level, but the principal component of any transport system - the human factor is still quite unexplored. The main obstacles are the lack of knowledge about the mental processes and the differences between the typical artificial intelligence approaches and the biological neural networks.

The artificial neural networks (ANN) seems to be a promising method for these models because the architecture of the ANN is directly inspired by the biological neuron and also because it is data driven method.

The classical paradigms of artificial neural networks are however not suitable for direct use because they simplify too much the real processes in biological neural network, but it is possible to update the learning algorithm so that it corresponds more frankly to the biological reality.

The neural networks have already been used in several projects aimed at mental models. One of them is the Blue Brain project [1, 2] with the goal to simulate the whole brain. This model should be so detailed that every cell is depicted on a molecular level. According to the authors, processes like consciousness, creativity, emotions or aggression will emerge.

Another research aimed at the models of human mind is the DARPA SyNAPSE project with opposite attitude. The idea behind this research is to create a neural network with architecture similar to biological neural network, however with strongly simplified neurons 3. The expectation is not to model mental processes, but to use the principles of biological neural network to improve the computer architecture. The way of information processing in the computer architecture is different from biological networks. In brain, the memory and computation are distributed; there is no central processor or memory. The expectation of SyNAPSE project is to understand the advantages of this architecture and to use them in formation of novel computer architecture.

With respect to both above mentioned researches, the target of this paper is to find such an equilibrium point where the model of the neuron is still faithful enough so that it can model the strong processes and 
yet be simple enough to be practically realizable.

\section{NeW SOLUTION - HOMEOSTATICAL NEURAL NETWORK}

The need for a compromise between the complexity of biological neural network and the practical feasibility of the artificial network led to a proposal of new learning algorithm. The idea is based on the classical multilayered neural network (MLP), the difference is in the learning process. The neurons are updating their parameters in a way that is similar to the real biological processes.

The basic idea is that the neurons are in competition for resources and the survival criterion is the usefulness of the neuron to the whole neural network. The neurons are not using any "teacher" or other kind of superior system, they have the same information as the biological neuron.

The learning process can be seen as searching of some equilibrium position which represents a state where the importance of the neuron for the neural network is maximal. This position can change in time if the environment changes.

The name of this type of learning, the homeostatic artificial neural network, is derived from the similarity of this idea to the process of homeostasis that is known from biology.

The proposed neural network is based on the idea of McCulloch-Pitts neuron:

$$
y=\phi f \sum_{i=0}^{n} x_{i} w_{i}
$$

where $f$ is the transfer function. The sigmoid transfer function was used:

$$
f(x)=\frac{2}{\left(1+e^{-\alpha * x}\right)-1}
$$

As the similarity to biological neuron was the basic requirement, the back propagation algorithm is not a solution because a higher structure (or teacher) is used to train the neuron. In the so called homeostatic neuron, the unit is using its proper forward connection to improve its function.

The 'axon' in this model has two functions - the first one is the transmission of the output to higher layer (as in back propagation), the second is the transmission of the utility information from higher layer to the lower one.

The idea of this type of training is that the neuron improves its relative importance in the network, in other words, it is trying to maximize the part of its output signal that is accepted by other neurons.

This idea corresponds to the biological reality because the information transmitted by the axon has the form of energy (and is inseparable from energy). Therefore, the neuron knows which part of its output energy was accepted by other neurons.
The process of learning can be described by the following algorithm: first, the neuron computes its output with its initial random weight. Then the neurons in the higher layer set their weights according to their level of contentment with the reference neuron. In the next step, the neuron changes its weights accordingly. Several possibilities of the weight change are described later in this paper. Then the new output is computed. The neurons in the higher layer read the output and re-calculate their inputs weights. The reference neuron then decides which setting was better.

This algorithm has several variants, all of them are using at least 2 successive values of the level of acceptance. This implies that the neuron must be equipped with a memory.

The process of learning is on figure 1

From the point of view of the reference neuron the learning is described by the following algorithm:

(1.) random initial weights

(2.) output with initial weights for the first input

(3.) neurons in higher layer (output neurons) compute the utility of the reference neuron and set their input weights accordingly. If they are satisfied with the output of the reference neuron, they increase their weights, otherwise they decrease them. There are several ways how to compute the utility, some of them are described in eq. 3- 6

(4.) the reference neuron changes one (or more) of its input weights

(5.) the reference neuron repeats the forward phase with the same data but with changed weight

(6.) the output neurons compute the utility (as in step 3)

(7.) the reference neuron evaluates the change in the step 4 - if it improves the utility, it will keep it, otherwise it will change the weights in the opposite direction

(8.) neuron repeats steps 2 to 7 with all the connections and all the inputs.

\section{CRiterions For optimization}

Several methods can be used for the calculation the importance of the neuron. The basic difference among them is the number of output neurons for which the reference neuron is 'working'.

The first extreme is a neuron that is optimizing its function for all output neurons. This neuron is 'reading' all output weights without taking any particular weight into consideration.

The opposite extreme is neuron that works only for one neuron in the higher layer; in other words is optimizing its function to improve its utility for this particular neuron. Apart from these options, many other compromise criterions can be defined. 
Set weights to initial random values

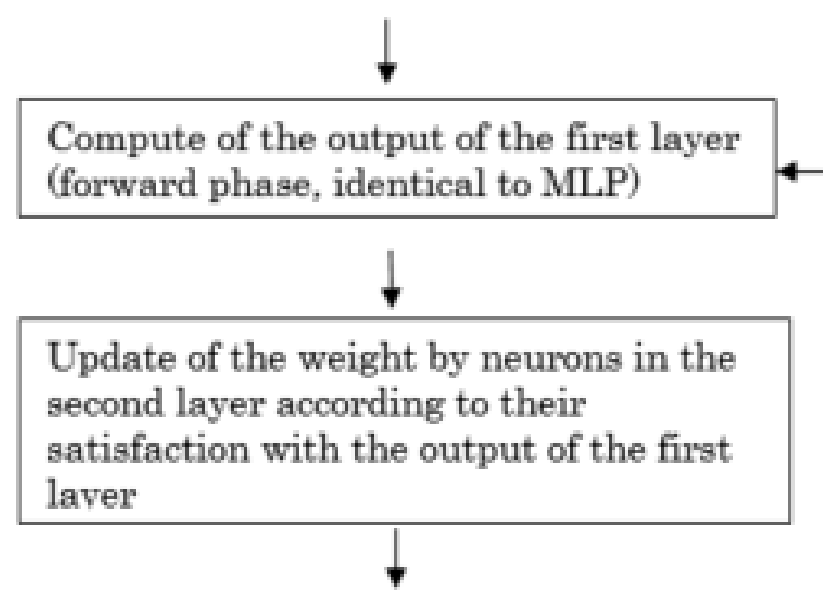

Utility computation by the neurons in the first layer. Value is stored to M1.

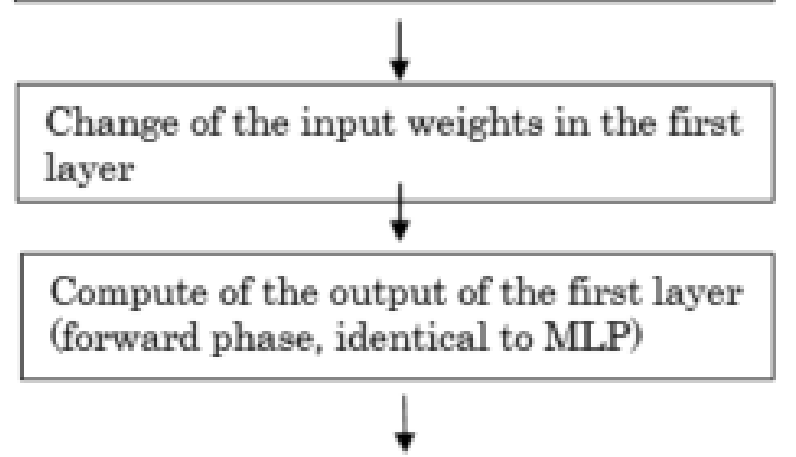

Update of the weight by neurons in the second layer according to their satisfaction with the output of the first

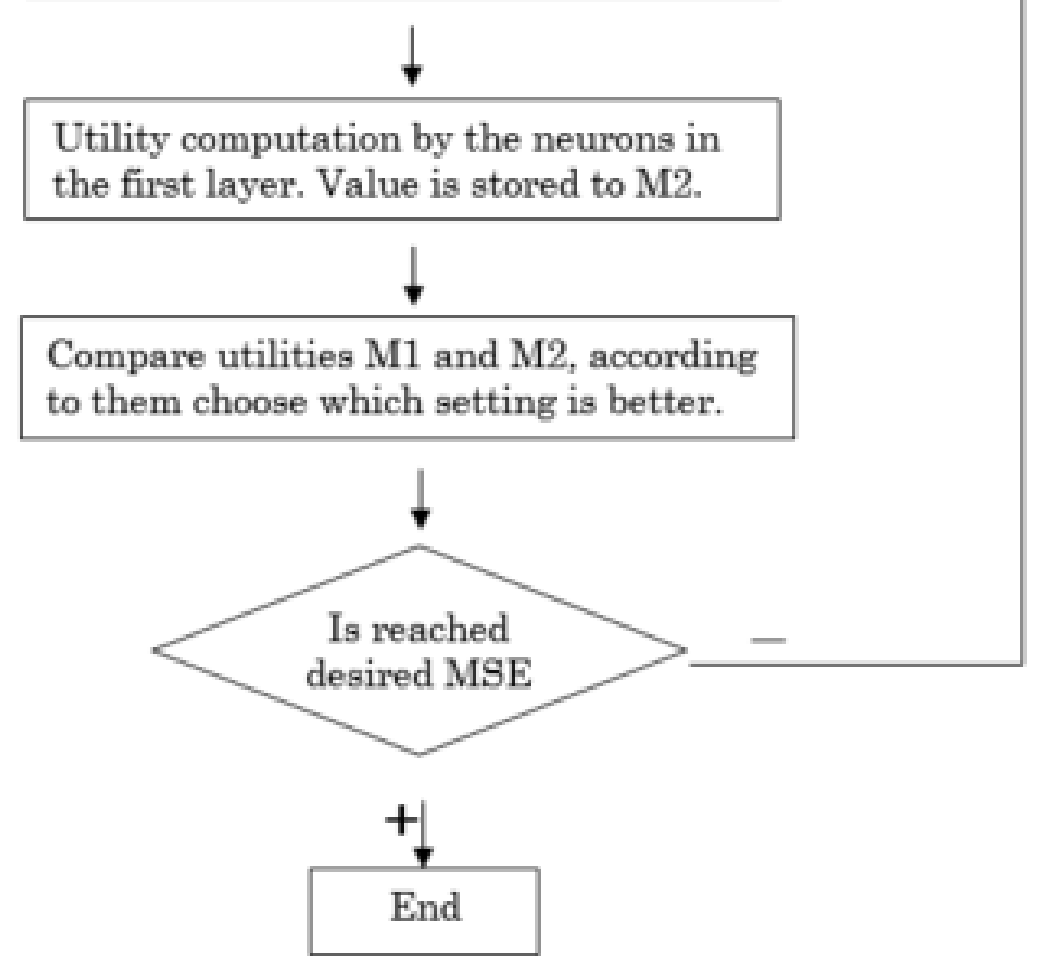

Figure 1. Wide figure [4, [5]. 
This first idea corresponds to a neuron that is finding such a weight vector $\wedge=w_{1}, w_{2}, \ldots, w_{n}$ for which the sum of the absolute values of the output weights is maximal. This idea corresponds firmly to the biological reality because the neuron has only one axon and therefore it can only be aware of the total amount of the signal that is accepted by other neurons, not of the particular weights.

In the case of the artificial neuron we also expect negative weights; therefore the neuron sums the absolute or square values. The utility $\mathrm{q}$ is:

$$
q=\sum_{j=1}^{n}\left|w_{j}^{o}\right|
$$

respectively:

$$
q=\sum_{j=1}^{n}\left(w_{j}^{o}\right)^{2}
$$

Eq. 4 puts stress on great values and reduces the importance of the small ones. This may be in certain cases advantage for the learning but does not correspond to the biological reality.

\section{Searching the one NeUron MAXIMUM - THE SECOND EXTREME}

The other type of training is based on the presumption that the neuron is increasing its importance to only one neuron in the higher layer, therefore it maximizes the function:

$$
q=\max \left|w_{j}^{o}\right| ; j \in[0,1, \ldots n]
$$

The problem is that if $\max \left|w_{o}\right|=1$, no further improvement is possible and the training stops. In a real situation, we expect networks with many neurons where this value will be reached very soon and then the learning stops. This is not the desired behavior; therefore in that case there should be used an additional condition that ensures the continuation of the training.

The solution of this problem is to use a compromise that takes into consideration more than one output neuron but not all of them. This can be done by optimization of some given number of maximal output weights:

$$
\begin{aligned}
& u=\max \left(\left|w_{o}\right|\right)+\max \left(w_{o}-\max \left(w_{o}\right)\right)+\ldots \\
& \text { where } w=\left\{w_{0}, \ldots, w_{n-1}\right\}
\end{aligned}
$$

\section{VARIANTS OF HOMEOSTATICAL NEURAL NETWORK}

Apart from the above mentioned variant, several other options must be defined.

First of all, the question of the weight range arises at least three options.
The most biologically plausible is to limit the weights to interval between 0 and 1 . That means that the weights cannot change the polarity of the signal and no gain of the output energy is allowed. This option is the most biologically plausible as the axon, synapse and dendrites are mostly working only as transmitters of the signal. On the other hand, some operation with the signal may be done also on the level of the axon - dendrit transmission, for example the inhibitory weights can reduce the neuron potential. This leads to the second idea, to limit the weights to interval $\langle-1 ; 1\rangle$.

The last option is not to limit the weights at all, that means the signal can be multiplied by any real number. This is the least biologically plausible method; on the other hand it will bring the highest computational power.

The other question is how to choose the weights that should be updated.

The first option that comes to mind is to update the weight that has the greatest influence on the result; that means the most sensitive weight. This will lead to highest increase of the utility of the neuron in the next step, however it may not be the best option from the global point of view. Making the step always in the direction of the highest gradient may lead to falling into local extreme. Also, to find the most sensitive weight consumes a lot of computational power.

Alternative option is to choose the updated weight randomly or in given order. These two options lead to similar results.

It is also possible to imagine the combination of these algorithms, for example by using several steps to update of the most sensitive weights which may be followed by randomly chosen weights.

Because of the complex nature of the neural networks, it is not possible to say which method is the best for arbitrary data.

\section{Problems of the idea of HOMEOSTATICAL LEARNING}

The described idea has two principal limitations.

The first one is the learning of the highest layer. The idea of a learning algorithm is that neurons in certain layers are updating their weights according to the higher layer. This can be used for all layers except for the highest as it does not have any output neuron.

The solution for practical simulation and testing is that the highest layer was not trained by the homeostatic learning algorithm, but by the back propagation. This is of course is an alteration of the original idea, but for the sake of the practical realization it is the easiest way how to program the homeostatic learning for the rest of the network. In the case of practical application, for example in robot, this problem will be solvable in a natural way because the network will be part of a closed loop.

The second problem is the delay question. The reference neuron is updating its input weights accord- 
ing to its output weights, but in each neural cell the output calculation takes some time., meanwhile the inputs are changing This means that the forward information will not 'meet' the information about the utility in the same time.

This problem has two solutions. The first is to set the dynamics of inputs to a lower level, so that the speed of information processing in the whole system is significantly higher than the changes of the input signals. The other possibility is to equip the neurons with a memory that stores the previous inputs so that it is possible to recall them when the information about the utility reaches the neuron.

\section{Conclusions}

In this paper, the idea of a new learning algorithm for neural networks is presented. This algorithm can cope with some disadvantages that the classical neural networks paradigms have. The proposed algorithm has several variants; from the point of view of the optimization criterion it is optimization for all input weights vs. optimization for only one input weights, and many compromising solutions.

From the point of view of the weight update, the possible variants are the update of the weight with the highest sensitivity to the change, of randomly chosen weight of consequently chosen weight.

From the point of view of the weight range, it is possible to define weights as positive numbers smaller than 1 , as either positive or a negative number in absolute value smaller than 1 , or as real numbers.

Every variant has its pros and cons. Due to the high number of variants which is even multiplied by the amount of data (for neural networks as for data driven method it is important which data is fed into the network. The same network can work well with one data and wrongly with another) it is difficult to decide which variants is the most promising. Larger testing is needed to understand the quality of the methods.

Despite the fact that the initial tests showed that the signal prediction task is better fulfilled by back propagation algorithm, the homeostatic neural network seems as a promising method for modeling on mental processes. Of course it is not possible to create a model of the complete consciousness, but it is possible to concentrate on some specific region. Transport engineering brings many possible applications as the human factor is the most important part in many transport systems. Several processes may be modeled and predicted by the use of this network. Typical example is a car driver, who is often making decisions with a lack of information and with the use of prior data. It is difficult to understand the factors that make for example the decision whether to cross the crossroads if the traffic light is orange and will become red in a short period of time. In such situations some drivers react differently even if the conditions are the same. The analytical way to explain such states is quite complex, but it is possible to collect enough data (either from real traffic or from a simulator) to predict this type of decisions. The neural network is a good tool to process this data.

Once the network is trained for certain tasks in a driver's decision making processes, it can be used for the investigation of other decisions and behavioral predictions. This can help to explain and avoid danger situations caused by aggressiveness, fatigue, emotions and others.

\section{REFERENCES}

[1] M. Růžek, T. Brandejský. Model of biological ANN based on homeostatic neurons. In 12th WSEAS International Conference on Neural Networks (NN'11), ISBN 978-960-474-292-9, Athens 2011, pp. 66 - 69.

[2] M. Růžek. Artificial Neural network inspired by homeostasis in biological networks. In Proceedings of 17th International Conference on Soft Computing (MENDEL 2011), ISBN 978-80-214-4302-0, pp. 232 - 235.

[3] M. Růžek. Artificial Neural Networks for Models of Driver's Brain Functions. In 20th Anniversary of the Faculty of Transportation Sciences, Czech Technical University in Prague - Selected Papers, ČVUT v Praze, 2013, ISBN 978-80-01-05320-1, pp. 207 - 211.

[4] M. Růžek. Modeling of EEG Signal with Homeostatic Neural Network. In Nostradamus 2013: Prediction, Modeling and Analysis of Complex Systems, Ostrava 2013, ISBN 978-3-319-00541-6 pp. 175 - 180.

[5] M. Růžek, T. Brandejský. Model of homeostatic artificial neuron. In Neural Networks, Fuzzy Systems \& Evolutionary Computing, ISBN 978-960-474-195-3, Athens 2010, pp. 145 - 148. 